\title{
Model of electrical conductivity of skeletal muscle based on tissue structure
}

\author{
F. L. H. Gielen \\ Department of Physies \& Astronomy, Vanderbilt University, Nashville, Tennessee, USA \\ H. E. P. Cruts \\ Research \& Innovation Group, Rehabilitation Centre Het Roessingh, Enschede, The Netherlands
}

\author{
B. A. Albers K. L. Boon W. Wallinga-de Jonge H. B. K. Boom \\ Department of Electrical Engineering, Twente University of Technology, PO Box 217 , \\ 7500 AE Enschede, The Netherlands
}

\begin{abstract}
Recent experiments carried out in our laboratory with the four-electrode method showed that the electrical conductivity of skeletal muscle tissue depends on the frequency of the injected current and the distance between the current electrodes. A model is proposed in order to study these effects. The model takes into account the structure of the tissue on the scale of individual fibres. It discerns three main components with respect to electrical properties: (a) extracellular medium with electrical conductivity $\sigma_{e}$, (b) intracellular medium with electrical conductivity $\sigma$; (c) muscle fibre membrane with impedance $Z_{m}$. The model results show an apparent frequency dependence of the electrical conductivity of skeletal muscle tissue, as well as the way the conductivity is affected by the length the current is conducted.
\end{abstract}

Keywords-Electrical conductivity, Skeletal muscle, Histological structure, Volume conduction, Model computation

Med. \& Biol Eng \& Comput., 1986, 24, 34,40

\section{Introduction}

ELECTRICAL CONDUCTIVITIES are important parameters in the description and interpretation of electrophysiological phenomena. In a recent paper (Gielen et al., 1984) we presented measurements of the electrical impedance of muscle tissue, carried out in our laboratory using the 'fourelectrode method'. In Fig. 1 a characteristic result of our measurements is shown together with values of conductivities of skeletal muscle tissue as reported by other authors (BURGER and VAN DONGEN, 1960; RUSH et al., 1963; EPSTEIN and Foster, 1983). Clearly the electrical conductivities as well as the values for the anisotropy $\left(=\sigma_{L} / \sigma_{T}\right)$ are frequency dependent in our measurements and in the report of EPSTEIN and FosTer (1983), whereas the other data from the literature are not, in the frequency range covered by EMG signals $(10 \mathrm{~Hz}-20 \mathrm{kHz})$.

In our experiments the interelectrode distance (IED) is only $0.05 \mathrm{~cm}$, which is at least ten times smaller than that used by other authors.

At such small IEDs the anatomical structure at the level of individual muscle fibres may become a factor that influences the measured electrical conductivity in a significant way. In order to study this aspect a model has been developed that describes the electrical conductivity of skeletal muscle tissue, taking into account the anatomical structure on the level of individual muscle fibres.

Correspondence should be addressed to Ir. B. A. Albers

First received 30th July and in final form 13th November 1984

(C) IFMBE: 1986

\section{Effective electrical conductivity}

A usual definition of the impedance $Z$ of a block of homogeneous medium is

$$
Z=\frac{L}{\sigma S}
$$

where $L$ is the length of the medium in the direction of the current, $S$ is the surface area of the medium in the direction perpendicular to the current, $\sigma$ is the complex electrical conductivity of the medium. If we define $Z^{\prime}$ as the impedance of a block of inhomogeneous media, the effective electrical conductivity of a block of inhomogeneous medium can be defined by

$$
\sigma_{e f f}=\frac{L}{Z^{\prime} S}
$$

with $Z^{\prime}=V / I$, where $V$ is the voltage difference on two opposite sides of a block of inhomogeneous medium with surface area $S$, and $I$ the total current in the direction normal to this surface. So with $\sigma_{\text {eff }}$ the relationship between the voltage difference on two opposite sides of a block of inhomogeneous medium and the current in the direction normal to this surface is given, but not the current distribution and potential field inside this block of inhomogeneous medium.

In the following Sections the effective electrical conductivity of muscle tissue in the directions parallel and transverse to the muscle fibres is derived from a model. We use a simplified representation of the muscle structure. 
The influence of connective tissues, blood (vessels) and nerves is neglected. We assume the muscle tissue contains only intra- and extracellular medium and muscle fibre membranes.

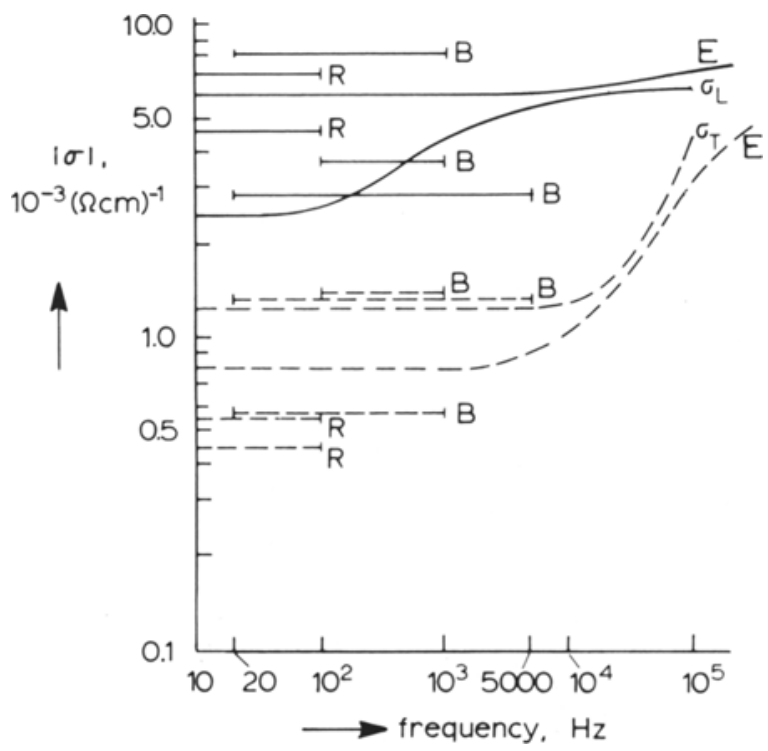

Fig. 1 Electrical conductivity $\sigma$ of skeletal muscle tissue obtained with the four-electrode method as a function of frequency. $B=B U R G E R$ and VAN DONGEN (1960); $R=$ RUSH et al. (1963); E = EPSTEIN and FOSTER (1983). The curves indicated with $\sigma_{L}$ and $\sigma_{T}$ are typical results of own measurements for the EDL-muscle in the hind limb of the rat in vivo (GIELEN et al., 1984). —— longitudinal; - - transverse

The structure assumed consists of small identical parts, called 'unit cells', all containing extracellular medium, intracellular medium and a part of the muscle fibre membrane.

In the following Sections we will define unit cells for the computation of the effective electrical conductivity of muscle tissue in directions both parallel and transverse to the muscle fibres.
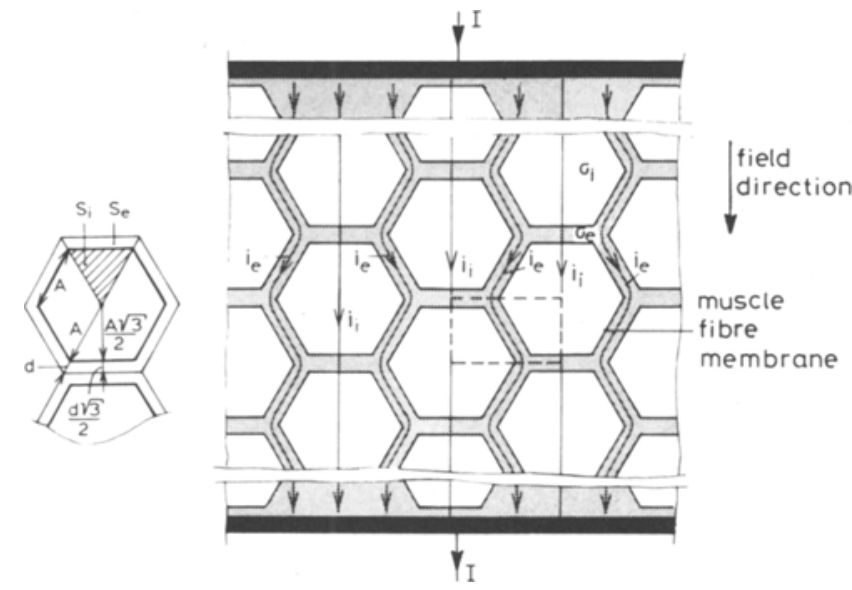

Fig. 2 Model structure used in the computation of electrical conductivity transverse to the muscle fibres. $I=$ the injected current; I divides into $i_{e}$, the extracellular component and $i_{i}$, the intracellular component; $S_{e}=$ the cross-sectional area of the extracellular compartment and $S_{i}=$ the cross sectional area of the intracellular compartment. $\sigma_{e}, \sigma_{i}=$ conductivities of the extra- and intracellular medium; $A=$ half diameter of the muscle fibres; $d=$ thickness of the layer of extracellular medium, surrounding the fibre. The broken lines show the geometry of the cross section of the unit cell, used for the computation

\section{Effective electrical conductivity transverse to muscle fibres}

In the calculation of the effective electrical conductivity transverse to the muscle fibres $\left(\sigma_{T M}\right)$, the structure of the muscle is simplified so as to consist of parallel hexagonal muscle fibres (see Figs. 2 and 4).

Current is impressed on the extracellular medium only, in a direction perpendicular to the muscle fibres (for instance by means of two large parallel conducting plates).

A unit cell for the computation of the electrical conductivity, transverse to the muscle fibres, is defined as a cell
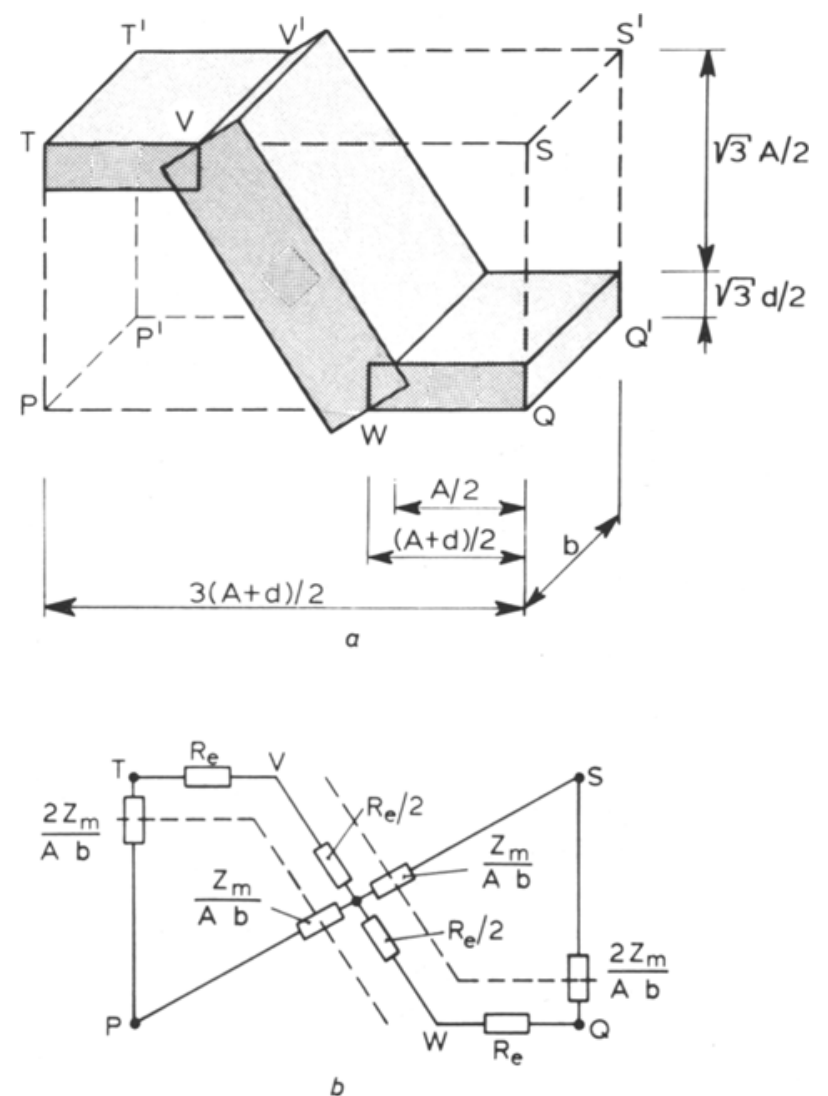

Fig. 3 (a) Assumed geometry of the unit cell used for the computation of $\sigma_{T M}$

(b) Lumped circuit equivalent of the unit cell (symbols are explained in the text)

with rectangular cross-section, shown with broken lines in Fig. 2. This unit cell is also shown in Fig. 3, together with a lumped circuit equivalent of the unit cell. In the calculation of the electrical impedance of this unit cell the following parameters are used (see Figs. 2 and 3):

$A=$ one half diameter of a muscle fibre (this means that the sides of the hexagonal cross section have a length $A$, see Fig. 2)

$d=$ distance given in Fig. 2, the thickness of the layer of extracellular medium between the fibres is $(3)^{1 / 2} d$ (we assume $d \ll A$, see Fig. 2)

$b=$ length of the unit cell parallel to the muscle fibres

$\sigma_{e}=$ conductivity of the extracellular medium

$\sigma_{i}=$ conductivity of the intracellular medium

$C_{m}=$ membrane capacity per unit of surface area

$G_{m}=$ membrane conductance per unit of surface area

$Z_{m}=$ impedance per unit of surface area

$p=$ fraction of the total volume of the tissue occupied by intracellular medium

$R_{e}=$ electrical resistance of the extracellular medium between $\mathrm{TT}^{\prime}$ and $\mathrm{VV}^{\prime}$, between $\mathrm{VV}^{\prime}$ and $\mathrm{WW}^{\prime}$, and between $\mathrm{WW}^{\prime}$ and $\mathrm{QQ}^{\prime}$ (see Fig. 3). 
Current flowing in a direction normal to the muscle fibre membrane meets with the series circuit of three impedances; namely the impedance of the intracellular medium, the membrane impedance and the impedance of the extracellular medium. If we compare the impedances of the intra- and extracellular medium with the much larger value of the membrane impedance for the frequency range concerned here, it is permissible to neglect these intra- and extracellular impedances.

The membrane impedance is approximated by a parallel circuit of $C_{m}$ and $G_{m}$. According to this approximation $1 / Z_{m}$ equals

$$
\frac{1}{Z_{m}}=G_{m}+j \omega C_{m}
$$

where $\omega=2 \pi f$ with $f$ the frequency and $j=(-1)^{1 / 2}$.

From eqn. 1 and Fig. $3 a$ the following relation is deduced for $R_{e}$ :

$$
R_{e}=\frac{A+d}{(3)^{1 / 2} \sigma_{e} d b}
$$

Fig. $3 b$ shows a lumped circuit equivalent for the unit cell of Fig. 3a. If, for example, the surfaces $\mathrm{PP}^{\prime} \mathrm{TT}^{\prime}$ and $\mathrm{QQ}^{\prime} \mathrm{SS}^{\prime}$ are two different equipotential surfaces, the modelled effective electrical conductivity $\sigma_{T M}$ of the unit cell can be computed using eqn. 1 and Fig. $3 b$. This results in

$$
\sigma_{T M}=\frac{(3)^{1 / 2}}{3 b R_{e}}+\frac{(3)^{1 / 2} A}{2 Z_{m}}
$$

If TT'SS' and $\mathrm{PP}^{\prime} \mathrm{QQ}^{\prime}$ are two different equipotential surfaces an identical result is obtained for $\sigma_{T M}$. Using eqn. 4 , eqn. 5 can be rewritten as

$$
\sigma_{T M}=\frac{d \sigma_{e}}{A+d}+\frac{(3)^{1 / 2} A}{2 Z_{m}}
$$

From Fig. $3 a$ it follows that the fraction of the total volume of the unit cell occupied by the intracellular medium equals

$$
p=\frac{A^{2}}{A^{2}+2 d A+d^{2}}
$$

With the assumption $d \ll A$ this results in

$$
\frac{d}{A}=\frac{1}{2}\left(\frac{1}{p}-1\right)
$$

A combination of eqns. 6 and 8 gives the effective electrical conductivity of the unit cell:

$$
\sigma_{T M}=\frac{1-p}{1+p} \sigma_{e}+\frac{(3)^{1 / 2} A}{2 Z_{m}}
$$

This formula shows that, due to the presence of $Z_{m}, \sigma_{T M}$ is frequency dependent.

\section{Effective electrical conductivity parallel to muscle fibres}

In the calculation of the electrical conductivity parallel to the muscle fibres $\left(\sigma_{L M}\right)$ the same simplified anatomical structure is used (see Fig. 4). Again the impedances of the intra- and extracellular medium for current flowing in a direction normal to the muscle fibre membrane are neglected. In this case current is impressed parallel to the muscle fibres. Now we take the unit cell as shown in Fig. $4 a$ (broken lines).
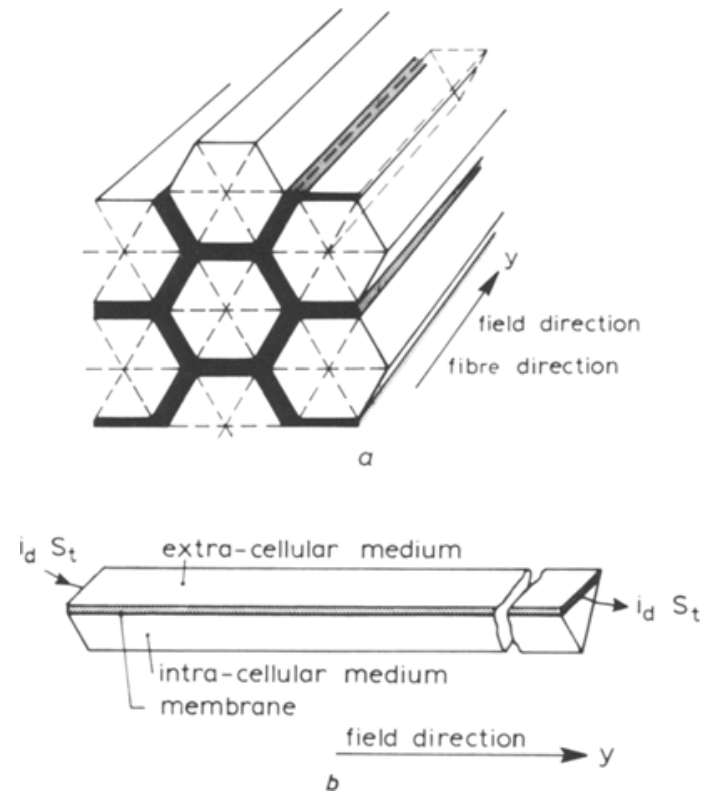

Fig. 4 (a) Model used in the computation of the electrical conductivity parallel to the muscle fibres

(b) Geometry of the unit cell

(symbols are explained in the text)

As before, current is impressed only on the extracellular medium, so current can only enter and leave the unit cell in the $y$-direction through the extracellular medium. This is shown in Fig. $4 b$.

Additional parameters used in the calculation of the effective electrical conductivity parallel to the muscle fibres are (see Fig. 5):

$$
\begin{aligned}
S_{t}, S_{e}, S_{i}= & \text { cross-sectional area of the total unit cell, the } \\
& \text { extracellular compartment in the unit cell and } \\
& \text { the intracellular compartment in the unit cell } \\
& \text { (see Fig. } 2 \text { ) } \\
= & \text { impressed current density (total current } \\
& \text { entering and leaving the unit cell equals } \left.i_{d} S_{t}\right)
\end{aligned}
$$

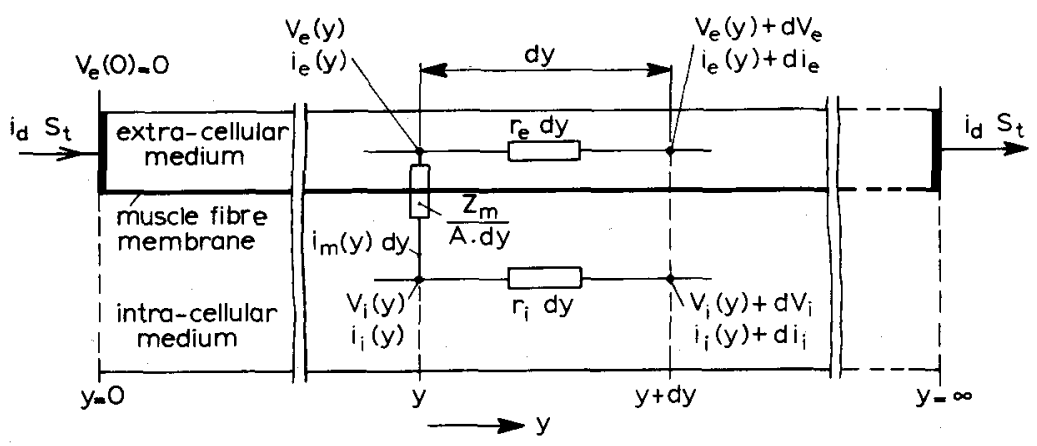


$V_{e}(y), V_{i}(y)=$ potentials in the unit cell in the extra- and intracellular medium, respectively, at position $y\left(\right.$ we choose $\left.V_{e}(0)=V_{i}(0)=0\right)$

$i_{e}(y), i_{i}(y)=$ current in the unit cell in the extra- and intracellular medium, respectively, at position $y$

$i_{m}(y) \quad=$ radial component of the current in the unit cell flowing through a segment of muscle fibre membrane per unit length

$r_{e}, r_{i}=$ longitudinal impedance of extra- or intracellular medium per unit length.

The impedance of a membrane segment with length $d y$ is equal to $Z_{m} / A d y$.

According to eqn. 1 the impedances $r_{e} d y$ and $r_{i} d y$ shown in Fig. 5 can be written as:

$$
\begin{aligned}
& r_{e} d y=\frac{1}{\sigma_{e}} \frac{d y}{S_{e}}=\frac{d y}{\sigma_{e}(1-p) S_{t}} \\
& r_{i} d y=\frac{1}{\sigma_{i}} \frac{d y}{S_{i}}=\frac{d y}{\sigma_{i} p S_{t}}
\end{aligned}
$$

Consider a part of the unit cell with length $d y$ at position $y$ (for the symbols used here, see Fig. 5). From Fig. 5 together with Ohm's law and the assumptions and definitions given above we derive the following differential equations:

$$
\begin{aligned}
\frac{d V_{e}(y)}{d y} & =-i_{e}(y) r_{e} \\
\frac{d i_{e}(y)}{d y}= & \frac{-\left(V_{e}(y)-V_{i}(y)\right) A}{Z_{m}} \\
= & \frac{-\left(V_{e}(y)\left(1+\frac{r_{i}}{r_{e}}\right)+r_{i} i_{d} S_{t} y-V_{0}\right) A}{Z_{m}}
\end{aligned}
$$

where $V_{0}$ is a constant.

Integration of eqns. 12 and 13 leads to:

$$
\begin{aligned}
& i_{e}(y)=B_{0} \frac{\lambda}{r_{e}} e^{\lambda y}-\lambda_{0} \frac{\lambda}{r_{e}} e^{-\lambda y}+\frac{r_{i}}{r_{e}+r_{i}} i_{d} S_{t} \\
& V_{e}(y)=-B_{0} e^{\lambda y}-\lambda_{0} e^{-\lambda y}-\frac{r_{e} r_{i}}{r_{e}+r_{i}} i_{d} S_{t} y+\frac{r_{e}}{r_{e}+r_{i}} V_{0}
\end{aligned}
$$

where

$$
\lambda=\left(\frac{A\left(r_{e}+r_{i}\right)}{Z_{m}}\right)^{1 / 2}
$$

and $B_{0}$ and $\lambda_{0}$ are constant. Due to the condition that the extracellular current $i_{e}(y)$ must have a finite value, $B_{0}=0$. The constants $V_{0}$ and $\lambda_{0}$ can be found using the following boundary conditions:

(i) $i_{e}(0)=i_{d} S_{t}$

(ii) $V_{e}(0)=0$

Elimination of the constants $V_{0}$ and $\lambda_{0}$ gives

$$
V_{e}(y)=\frac{-i_{d}}{\sigma_{e}(1-p)+\sigma_{i} p}\left(y+\frac{1-e^{-\lambda y}}{\lambda} \frac{\sigma_{i} p}{\sigma_{e}(1-p)}\right)
$$

According to eqn. $2 \sigma_{L M}$ can be written as

$$
\sigma_{L M}=\frac{1}{Z^{\prime}} \frac{L}{S}
$$

$$
=\frac{-i_{d}}{V_{e}(y)} y
$$

Substitution of eqn. 19 in eqn. 20 results in the following expression for $\sigma_{L M}$ :

$$
\sigma_{L M}=\frac{\sigma_{e}(1-p)+\sigma_{i} p}{1+\frac{1-e^{-\lambda y}}{\lambda y} \frac{\sigma_{i} p}{\sigma_{e}(1-p)}}
$$

where

$$
\lambda=\left(\left(\frac{1}{\sigma_{e}(1-p) S_{t}}+\frac{1}{\sigma_{i} p S_{t}}\right) \frac{A}{Z_{m}}\right)^{1 / 2}
$$

So $\sigma_{L M}$ depends on frequency as well as on $\lambda y$, and hence $\sigma_{L M}$ is different from the electrical conductivity usually encountered. As a consequence the electrical properties of muscle tissue cannot be characterised by means of one longitudinal electrical conductivity, independent of measuring geometry.

The value of $\lambda y$ characterises three ranges of $\sigma_{L M}(\lambda y)$ values :

(a) $|\lambda y|<0 \cdot 1$

Eqn. 21 reduces to

$$
\sigma_{L M}(\lambda y) \approx \sigma_{e}(1-p)
$$

The current flows almost exclusively in the extracellular medium. In this case the unit cell can be replaced by an identical cell of a homogeneous medium with conductivity $\sigma_{L L M}=\sigma_{e}(1-p)$.

$$
\begin{aligned}
\text { For }|\lambda y|<0.1 \\
0.95<\left|\frac{\sigma_{L L M}}{\sigma_{L M}(\lambda y)}\right|<1.00
\end{aligned}
$$

(b) $|\lambda y|>100$

Eqn. 21 reduces to

$$
\sigma_{L M}(\lambda y) \approx \sigma_{e}(1-p)+\sigma_{i} p
$$

Now current flows in the intra- as well as in the extracellular medium depending on the conductivity of the intraand extracellular medium. The unit cell can be replaced by an identical cell of a homogeneous medium with conductivity $\sigma_{L H M}=\sigma_{e}(1-p)+\sigma_{i} p$.

$$
\text { For }|\lambda y|>100
$$

$$
1.00<\left|\frac{\sigma_{L H M}}{\sigma_{L M}(\lambda y)}\right|<1.05
$$

(c) $0 \cdot 1<|\lambda y|<100$

In this case eqn. 21 cannot be simplified, implying that the current distribution depends on the value of $\lambda y$. Close to the current source (or sink) current flows in the extracellular medium only. Far from the source (or sink) current flows in the intra- as well as in the extracellular medium.

The distribution of current depends on the conductivities of these media and on the impedance of the muscle fibre membrane, and is therefore frequency dependent.

We conclude that according to the model the current distribution depends on the distance between the (electrophysiological) source and the position of the measuring point as well as on the frequency contents of the signal, generated by the source. 


\section{Numerical results}

In Fig. 6 the effect of changing the interelectrode distance from $0.05 \mathrm{~cm}$, used in our experiments (GIELEN et al., 1984), to a value of the same order of magnitude as the interelectrode distance mentioned by Rush et al. (1963) $(>0.5 \mathrm{~cm})$ is illustrated. The values are calculated for a situation comparable with the four-electrode method, but with infinite large current electrodes. The parameter values used in the computation of $\sigma_{T M}$ and $\sigma_{L M}$ are given in the legend to Fig. 6. The results show that the interelectrode distance influences the degree of frequency dependence of the effective electrical conductivities. At frequencies less than $1 \mathrm{kHz}$ for large interelectrode distances the values obtained for the conductivity parallel to the muscle fibres are more than twice the conductivity values obtained with small interelectrode distances.

\section{Discussion}

The model presented takes into account the anatomical structure of skeletal muscle tissue on the scale of individual muscle fibres. It predicts that the conductivity of electric current by skeletal muscle tissue depends on the frequency contents of the injected current and that the geometrical scale, i.e. the distance between the site of current injection
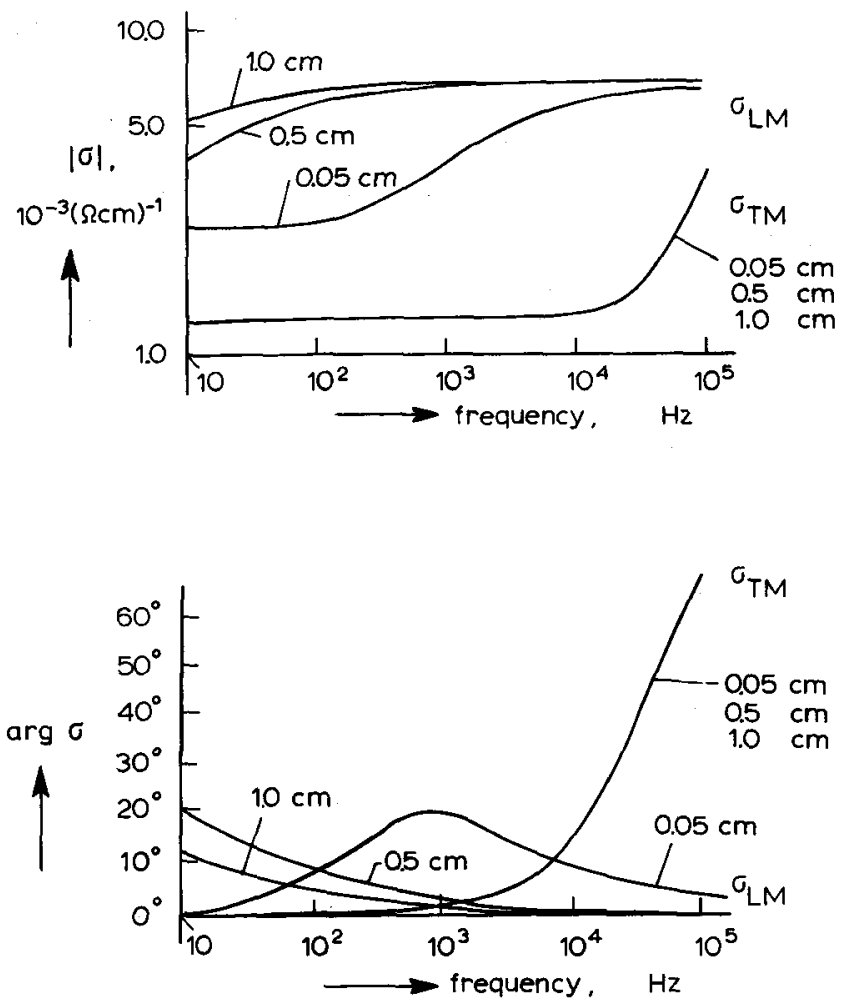

Fig. 6 Computed electrical conductivities $\left(\sigma_{L M}(I E D)\right.$ and $\sigma_{T M}$ $(I E D))$ at different interelectrode distances $(I E D=0.05 \mathrm{~cm}$, $0.5 \mathrm{~cm}$ and $1.0 \mathrm{~cm}$ ). The following parameter values were used: $\sigma_{e}=2.4 \times 10^{-2}(\Omega \mathrm{cm})^{-1} ; \sigma_{i}=5.5 \times 10^{-3}$ $(\Omega \mathrm{cm})^{-1} ; \quad G_{m}=1.0 \times 10^{-4}\left(\Omega \mathrm{cm}^{2}\right)^{-1} ; \quad C_{m}=1.0$ $\times 10^{-6} \mathrm{~F} \mathrm{~cm}^{-2} ; p=0.9 ; A=25 \times 10^{-4} \mathrm{~cm}$

and the location of the voltage measurement, influences the value of the conductivity in the direction parallel to the fibre direction.

Such model predictions have been done earlier in other fields. For the impedance of cerebral white matter, with a fibrous structure, NichOLSON (1965) deduced a formula (p. 398, eqn. 11) that is essentially the same as eqn. 9 in this paper. Nicholson used the theory for diluted suspensions (MAXWELL, 1896) as a starting point for his study. V.
OOSTEROM et al. (1979) used a formula describing the electrical conductivity of a suspension of parallel cylinders. HaAs and Brommundt (1980) derived a formula for the electrical conductivity of a bundle of fibres (from cardiac or smooth muscle) with intracellular clefts. Their formula is primarily based on the anatomical structure of the tissue, as it is in this paper. They modelled the tissue with parallel nonconducting fibres and derived a description of the transverse conductivity essentially the same as eqn. 6 in which the membrane impedance has an infinitely high value.

However, no description, taking into account the anatomical structure and the electrical properties of the components of the tissue, of the electrical conductivity parallel to skeletal muscle fibres has been reported in the literature.

Direct confirmation of the model results requires measurement of the electrical potential in the muscle, as caused by the injection of current by means of large, flat and essentially extracellular electrodes. Usually, however, measurements of conductivity are based on the four-electrode method: a potential difference caused by current injection by two point-shaped electrodes is measured. Despite the conceptual differences between the model presented and the experimental situation in which conductivity data are usually obtained (GIELEN et al., 1984), there is a good agreement between computed and measured effective electrical conductivities. It is as yet uncertain if this agreement allows the conclusion that the shape of the current electrodes has little influence on the conductivity values. For a correct comparison, the model should be adapted so that it can describe potential fields generated by point-shaped current electrodes. With the model presented, this appears to be unattainable.

Recently Plonsey and BARR (1982) analysed the fourelectrode method for homogeneous bidomain media. In this approach it was supposed that current is redistributed between intra- and extracellular space, thought to be continuously mixed. The authors achieved an analytical solution for the intra- and extracellular potential field, assuming equal ratios of the intra- and extracellular conductivities in the three principal directions. When the bidomain analysis of Plonsey and Barr is applied to the case of one-dimensional current field, as in our model study, the result is a one-dimensional equivalent of their bidomain model:

$$
V_{e}(y)=\frac{-I}{g_{e y}+g_{i y}}\left(y+\frac{1-e^{-\gamma y}}{\gamma} \frac{g_{i y}}{g_{e y}}\right)
$$

where

$g_{e y}, g_{i y}=$ conductivities defined on the total space occupied by the extracellular and intercellular medium, respectively

$$
\begin{aligned}
& \gamma=\left(B\left(\frac{1}{g_{e y}}+\frac{1}{g_{i y}}\right) / Z_{m}\right)^{1 / 2} \\
& B=\text { surface/volume ratio of fibres } \\
& Z_{m}=\frac{1}{G_{m}+j \omega C_{m}} \text { membrane impedance per unit } \\
& \text { length (see Section 3). }
\end{aligned}
$$

Eqn. 25 can be compared with our eqn. 19 for the longitudinal case. Both formulas would be identical if and only if the following relations existed between their parameters:

$$
B=\frac{A}{S_{t}}
$$




$$
\begin{aligned}
& g_{i y}=\sigma_{i} p \\
& g_{e y}=\sigma_{e}(1-p)
\end{aligned}
$$

In the $x$ - and $z$-direction (transverse directions) the same functional relationship (eqn. 25) exists in the model approach of Plonsey and Barr. However, in our model, no redistribution of current takes place in the transverse direction, making the models differ as to their dependence on the transverse co-ordinates $x$ and $z$. It may be of interest in how far both models correspond if we take distances at which, using the model of Plonsey and Barr, redistribution of current reaches its steady value. From eqn. 25 in the $x$-direction for $x y \gg 1$

$$
V_{e}(x)=\frac{-I x}{g_{e x}+g_{i x}}
$$

From our model, a formula for the extracellular potential can be derived, using eqn. 9 for the effective transverse electrical conductivity:

$$
V_{e}(x)=\frac{-I x}{\sigma_{T M}}=\frac{-I x}{\frac{1-p}{1+p} \sigma_{e}+\frac{(3)^{1 / 2} A}{2\left(Z_{m}+R_{i n}\right)}}
$$

An additional intracellular resistance $R_{i n}$ normal to the muscle fibre membrane is used; $R_{\text {in }}=\left((3)^{1 / 2} A\right) /\left(2 \sigma_{i}\right)$. This parameter is required as we will examine high-frequency behaviour. For high frequencies the membrane impedance will vanish and eqn. 30 becomes

$$
V_{e}(x)=\frac{-I x}{\frac{1-p}{1+p} \sigma_{e}+\sigma_{i}}
$$

This formula will be identical with eqn. 29 when the following relations exist:

$$
\begin{aligned}
& g_{e x}=g_{e z}=\frac{1-p}{1+p} \sigma_{e} \\
& g_{i x}=g_{i z}=\sigma_{i}
\end{aligned}
$$

As mentioned earlier, Plonsey and Barr assumed equal ratios of the intra- and extracellular conductivities in the three principal directions. Eqns. 27 and 28, together with eqns. 32 and 33 , show that this assumption, which could be valid in a one-dimensional equivalent of the bidomain model, is in fact in conflict with the geometrical assumptions underlying our model.

An important aspect in the model presented is the redistribution of the current injected into the muscle tissue. This will also occur in the case of current injection caused by an excited muscle fibre membrane. The resulting extracellular action potential will be influenced by this current redistribution. The results presented suggest that volume conduction models aimed at giving a correct description of electrophysiological signals should be adapted in a way that the anatomical structure on the scale of individual muscle fibres is taken into account.

Acknowledgement-This work has been supported by the Netherlands Organisation for the Advancement of Pure Research (ZWO).

\section{References}

Burger, H. C. and van Dongen, R. (1960) Specific electric resistance of body tissues. Physics in medicine and biology, 5, 431447.
EPSTEIN, B. R. and FosteR, K. R. (1983) Anisotropy in the dielectric properties of skeletal muscle. Med.\& Biol. Eng. \& Comput., 21, 51-55.

Gielen, F. L. H., Wallinga-de Jonge, W. and Boon, K. L. (1984) The electrical conductivity of skeletal muscle tissue: experimental results from different muscles in vivo. Med. \& Biol. Eng. \& Comput., 22, 569-577.

HAAS, H. G. and BrommundT, G. (1980) Influence of intercellular clefts on potential and current distribution in a multi fiber preparation. Biophys. J., 30, 327-350.

MAXWELL, J. C. (1896) A treatise on electricity and magnetism, 3rd edn. Dover, New York.

Nicholson, P. W. (1965) Specific impedance of cerebral white matter. Experimental neurology, 13, 386-401.

Oosterom, A., van de Boer, R. W. and VAN Dam, R. Th. (1979) Intramural resistivity of cardiac tissue. Med. \& Biol. Eng. \& Comput., 17, 337-343.

Plonsey, R. and BARR, R. (1982) The four-electrode resistivity technique as applied to cardiac muscle. IEEE Trans., BME29/7, 541-546.

Rush, S., Ablldskov, J. A. and McFee, R. (1963) Resistivity of body tissues at low frequencies. Circulation research, 12, 40-50.

\section{Authors' biographies}

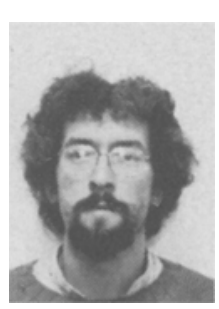

Frans L. H. Gielen was born in Emmeloord, The Netherlands, in 1951. He received the B.A. degree in Physics (Cryogenics) from Twente University of Technology in 1976, and the M.Sc. degree in Physics (Biophysics) from the same university in 1979. His Ph.D. research was granted by the Dutch Society for Scientific Research (ZWO-Biofysika) and was performed between 1979 and 1983 in the Biomedical Engineering Group of the Department of Electrical Engineering of Twente University of Technology. His special interests are electrophysiology and electric volume conduction theory with respect to EMG signals. $\mathrm{He}$ joined Department of Physics \& Astronomy of Vanderbilt University, Nashville, Tennessee, USA in February 1984 where his research is on electric and magnetic measurements and models based on electromagnetic theory, which are used for the interpretation of experimental results

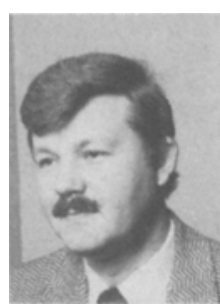

Kasper L. Boon was born in Amsterdam, The Netherlands, in 1944. He received an M.Sc. degree in Physics in 1967 from the Technical University of Eindhoven and a Ph.D. in Biomedical Engineering from the Twente University of Technology in 1974 . He is a senior staff member of the Biomedical Engineering Group, Department of Electrical Engineering of the Twente University of Technology. His research activities are mainly directed towards electromyography.

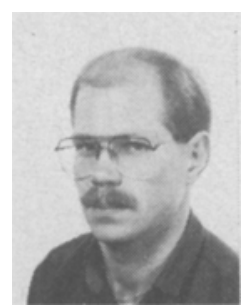

Hubert E. P. Cruts was born in The Netherlands, in 1958. He received the M.S. degree in electrical engineering from the Twente University of Technology, Enschede, The Netherlands in 1982 . He is presently associated with the Research and Innovation Group, Rehabilitation Centre Het Roessingh, Enschede, The Netherlands. His research interests are in biomedical signal processing with application to exercise-ECG analysis.

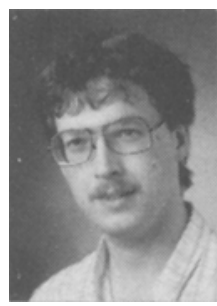

Bert A. Albers was born in Hengelo, The Netherlands, in 1958. He received his M.Sc. degree in Physics from Twente University of Technology in 1983. In the same year he joined the Biomedical Engineering Division, Department of Electrical Engineering, of the Twente University of Technology. His research interests are electrophysiology and volume conduction in skeletal muscle tissue. 


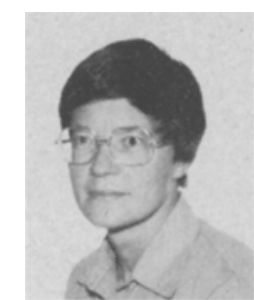

Willemien Wallinga-de Jonge was born in Veendam, The Netherlands, in 1945. She received the M.Sc. degree in Biology from the University of Groningen in 1968 and her Ph.D. in Biomedical Engineering from Twente University of Technology, Enschede, in 1980.

She is a senior staff member of the Biomedical Engineering Group, Department of Electrical Engineering of Twente University of Technology. Her research activities there involve the electrical activity of muscle in general and the intracellular and extracellular single fibre activity in particular.

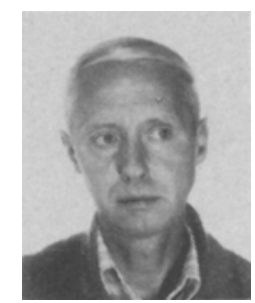

Herman B. K. Boom was trained as a medical physicist at the University of Utrecht, where he received his Ph.D. in 1971. He joined the Departments of Medical Physics and Medical Physiology where he was engaged in research in the field of cardiac mechanics and taught physiology and biophysics. Since 1976 he has occupied the Chair of Medical Electronics in the Electrical Engineering Department of Twente University of Technology. His research interests are cardiovascular system dynamics, bioelectricity and rehabilitation technology. 